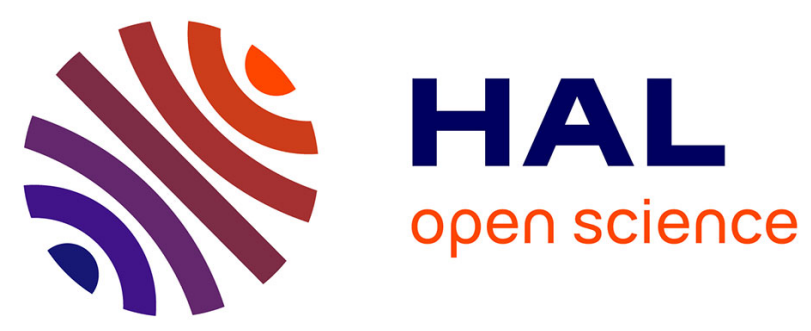

\title{
Les langues de l'Angleterre médiévale : au-delà du bilinguisme
}

\author{
Alban Gautier, Jean-Pascal Pouzet
}

\section{To cite this version:}

Alban Gautier, Jean-Pascal Pouzet. Les langues de l'Angleterre médiévale : au-delà du bilinguisme. Médiévales, 2015, 68, pp.15-24. 10.4000/medievales.7473 . hal-02131209

HAL Id: hal-02131209

https://hal-normandie-univ.archives-ouvertes.fr/hal-02131209

Submitted on 16 May 2019

HAL is a multi-disciplinary open access archive for the deposit and dissemination of scientific research documents, whether they are published or not. The documents may come from teaching and research institutions in France or abroad, or from public or private research centers.
L'archive ouverte pluridisciplinaire HAL, est destinée au dépôt et à la diffusion de documents scientifiques de niveau recherche, publiés ou non, émanant des établissements d'enseignement et de recherche français ou étrangers, des laboratoires publics ou privés. 


\section{Médiévales}

Langues, Textes, Histoire

68 | printemps 2015

Langues d'Angleterre

\section{Les langues de l'Angleterre médiévale : au-delà du bilinguisme}

The Languages of Medieval England: Beyond Bilingualism

Alban Gautier et Jean-Pascal Pouzet

\section{(2) OpenEdition}

Journals

Édition électronique

URL : http://journals.openedition.org/medievales/7473

DOI : $10.4000 /$ medievales.7473

ISSN : 1777-5892

Éditeur

Presses universitaires de Vincennes

Édition imprimée

Date de publication : 15 juin 2015

Pagination : 15-24

ISBN : 978-2-84292-430-0

ISSN : 0751-2708

Référence électronique

Alban Gautier et Jean-Pascal Pouzet, « Les langues de l'Angleterre médiévale : au-delà du bilinguisme », Médiévales [En ligne], 68 I printemps 2015, mis en ligne le 15 juin 2017, consulté le 08 mai 2019.

URL : http://journals.openedition.org/medievales/7473; DOI : 10.4000/medievales.7473 



\section{Alban Gautier et Jean-Pascal Pouzet}

\section{Les langues de l'Angleterre médiévale: au-delà du bilinguisme}

Quand on est deux, l'hymen est une chaîne Dont il est malaisé de supporter le poids; Mais on la sent peser à peine Quand on est trois ${ }^{1}$.

À première vue, la question des langues de l'Angleterre médiévale pourrait sembler rebattue, et la présente entreprise quelque peu périlleuse. Il est vrai que plusieurs travaux récents proposent des états des lieux, des analyses ou des synthèses sur telle ou telle partie de cette période de quelque dix siècles ${ }^{2}$. C'est que, tout au contraire, la relative vitalité de la recherche en ce domaine signale que le sujet des langues en contact dans l'espace anglais médiéval est loin d'être épuisé, et que certaines facettes, encore mal explorées, ont besoin pour mieux l'être d'un surcroît d'approche transdisciplinaire, ainsi que le reconnaît également Elizabeth Tyler ${ }^{3}$.

1. H. Meilhac et L. Halévy, Livret de La Belle Hélène, musique de Jacques Offenbach, cité d'après A. PÂris éd., Livrets d'Opéra, Paris, 1991, vol. I: De Beethoven à Purcell, p. 9791017 (p. 994).

2. Sur les vingt-cinq dernières années, citons entre autres T. Hunt, Teaching and Learning Latin in Thirteenth-Century England, 3 vol., Cambridge, 1991; D. TROTTER éd., Multilingualism in Later Medieval Britain, Woodbridge, 2000; J. SCAHILL, «Trilingualism in Early Middle English Miscellanies: Languages and Literature», Review of English Studies, 33 (2003), p. 18-32; E. M. TYLER éd., Conceptualizing Multilingualism in England, 8001250, Turnhout, 2011.

3. E. M. TYLER, «Introduction. England and Multilingualism, Medieval and Modern», dans EAD. éd., Conceptualizing Multilingualism..., p. 1-13 (p. 12): «While multilingualism poses a challenge to disciplinary formations and insists on greater permeability between them, this challenge can be met by collaborative work which builds on the varied foundations of our disciplines and combines our different linguistic and chronological expertises to ask new questions of the past. » 
Voici précisément l'une des deux raisons d'être de la présente publication et de l'atelier qui l'a préparée à Poitiers en septembre $2013^{4}$ : la rencontre de spécialistes d'histoire, de philologie, de littérature et de certaines des langues de cet espace afin d'aborder de front, et pour un lectorat francophone, un phénomène aussi diffus que protéiforme, que nulle discipline isolément prise ne saurait circonscrire. Dans cette interaction fructueuse entre les disciplines, mais restreinte ici par nécessité à une poignée de chercheurs, aucune prétention à l'exhaustivité ne pouvait évidemment être de mise. On verra aisément que les langues présentes sous une forme ou une autre dans l'Angleterre médiévale sont diversement couvertes, avec une prédominance (sans doute assez inévitable pour un dossier limité à la période suivant la Conquête normande) de ce qu'il faut bien reconnaître, toutes situations confondues, comme les trois principales: le latin, diffusé dans l'île avant même la création d'une province de Bretagne sous le règne de l'empereur Claude au I Ir siècle de notre ère; l'anglais, né en Britannia au cours des siècles «obscurs» (en termes documentaires) que sont les $\mathrm{V}^{\mathrm{e}}$ et $\mathrm{VI}^{\mathrm{e}}$ siècles; le français d'Angleterre (ou anglo-normand, ou anglo-français), présent à partir des dernières décennies du $\mathrm{XI}^{\mathrm{e}}$ siècle. À cette prédominance se mesurent l'absence ou la discrétion de certaines autres langues dans les pages qui suivent: par exemple, en amont de la formation de ce trio, les langues brittoniques refoulées par l'anglais vers les franges occidentales de l'île, l'irlandais des missionnaires «scots» venus d'Iona au vII siècle, le grec introduit à la fin du même siècle à Cantorbéry par l'archevêque Théodore et l'abbé Hadrien, ${ }^{5}$ ou encore le norrois apporté par les migrants scandinaves entre le $\mathrm{IX}^{\mathrm{e}}$ et le $\mathrm{XI}^{\mathrm{e}}$ siècle; mais aussi, au lendemain de la Conquête, les langues scandinaves et germaniques dont la diversité colorait

4. Les articles ici rassemblés ont été présentés lors de l'atelier préparatoire «Les langues de l'Angleterre médiévale: au-delà du bilinguisme », qui s'est tenu le 13 septembre 2013 au Centre d'études supérieures de civilisation médiévale. Nos remerciements vont d'abord au CESCM, tout particulièrement à sa directrice, Cécile Treffort, et à son directeur adjoint, Stephen Morrison, pour leur accueil, ainsi qu'à Laurent Boutin, Catherine Giraud, Nicole Henry et Élisabeth Nau pour l'aide apportée dans la préparation et la tenue de la rencontre. Nous remercions également le CNRS, l'Université de Poitiers (UFR Lettres et Langues), le laboratoire HLLI de l'Université du Littoral-Côte d'Opale (équipe CRHAEL), l'Institut universitaire de France et le GDR «Mondes britanniques», qui ont épaulé techniquement ou financièrement cet atelier. La journée préparatoire a réuni sept participants : pour diverses raisons, quatre seulement ont été en mesure de soumettre un texte, ce qui a permis la publication de ce dossier. On lira un compte rendu de l'atelier, avec un résumé des communications manquantes (celles de Jean-Pascal Pouzet, Emmanuelle Roux et Olivier Szerwiniack), sur le site de l'équipe CRHAEL, sous l'onglet «Ateliers et séminaires » (http:// crhael.univ-litttoral.fr/?page_id=249).

5. M. C. BodDEN, «Evidence for Knowledge of Greek in Anglo-Saxon England», Anglo-Saxon England, 17 (1988), p. 217-246. 
l'atmosphère des ports de la mer du Nord ${ }^{6}$, ou le flamand des mercenaires au service des rois normands ${ }^{7}$; et enfin, à Londres à partir du XIV e siècle, le flamand toujours, celui d'artisans immigrés de première génération ou d'artistes de passage ${ }^{8}$, ou l'italien plus éphémère de marchands en poste ou de passage pour leurs affaires. On verra néanmoins se glisser, comme dans les interstices du trio de tête, quelques autres idiomes parlés ou écrits dans l'Angleterre des siècles médiévaux: l'hébreu manié par les communautés juives installées dans le royaume au lendemain de la Conquête ${ }^{9}$; le gallois d'anthroponymes rapportés dans les documents d'archive ${ }^{10}$. N'oublions pas, en outre, que le français et l'anglais eux-mêmes se manifestent à l'occasion dans toute leur diversité dialectale. Mais la dissymétrie du traitement des langues se rachète un peu, nous l'espérons, par l'originalité des réflexions et la nouveauté de certaines pistes qui s'esquissent dans ces pages.

La seconde raison de cette publication, c'est qu'il a semblé qu'il pouvait y avoir un intérêt particulier à privilégier des situations précises d'interaction: celles pour lesquelles plus de deux langues sont impliquées. De fait, les instances de phénomènes multilingues le plus souvent étudiées dans les travaux existants sont bien celles qui impliquent la rencontre entre deux langues: en général le latin et l'anglais, le latin et le français, le français et l'anglais ${ }^{11}$. Au contraire, l'importance du trilinguisme paraît encore un peu minorée dans les différents domaines de la réflexion. Pour ne prendre qu'un seul exemple, il n'existe presque aucune «triangulation» dans la réflexion portant sur la traduction, cette langue aventurée au possible d'une autre langue, si l'on nous permet de paraphraser la belle expression d'Édouard Glissant ${ }^{12}$. Notre exergue emprunté à Offenbach n'est donc pas entièrement incongru. L'enlèvement de l'Hélène offenbachienne n'est-elle pas une figure, même parodique, de la translatio imperii et studii? Le rapport

6. F. LENÈGRE, «Norvégiens et Britanniques : identités et altérité au sein d'une Méditerranée septentrionale (XII ${ }^{e}$-XIV ${ }^{e}$ siècle)», dans A. GAUTIER et S. Rossignol éd., De la mer du Nord à la mer Baltique. Identités, contacts et communications au Moyen Âge, Villeneuved'Ascq, 2012, p. 205-220.

7. E. OKSANEN, Flanders and the Anglo-Norman World, 1066-1216, Cambridge, 2012.

8. S. CASSAGNE-BRouQueT, «Les artistes flamands à Londres: échanges culturels ou conquête d'un marché?», dans Les Échanges culturels au Moyen Âge. Actes du XXXII ${ }^{e}$ congrès de la SHMESP, Paris, 2002, p. 283-292.

9. C. Rотн, A History of the Jews in England, $3^{e}$ éd., Oxford, 1964, p. 4-6. Voir cidessous l'article de D. Trotter.

10. Ci-dessous dans l'article de C. Nall et D. Wakelin.

11. Par exemple I. SHORT, «'Tam Angli quam Franci': Self-Definition in Anglo-Norman England», Anglo-Norman Studies, 18 (1996), p. 153-175.

12. É. Glissant, Poétique de la Relation, Paris, 1990, p. 130: «L'apprentissage et la traduction ont ceci de commun qu'ils tentent de redonner au texte "de la transparence". C'està-dire qu'ils s'efforcent de jeter un pont entre deux séries d'opacités: d'un texte opposé à un lecteur novice pour qui tout texte est réputé difficile (c'est le cas de l'apprentissage), d'un texte aventuré au possible d'un autre texte (c'est le cas de la traduction). » 
des langues relève semblablement de cette problématique de la translatio, et se concentrer sur deux langues serait notoirement insuffisant. Dans un cadre plus «léger», plus dynamique, les interférences s'éclairent mieux à trois que dans le face à face bloqué de deux langues affrontées ce qui ne veut pas dire moindre complexité, bien au contraire. Mais à trois seulement car, pour l'essentiel, «au-delà du bilinguisme» renvoie à des situations de mise en présence de trois langues, et pas plus: les cas de «quadrilinguisme» sont fort limités, non attestés à notre connaissance pour la communication orale, très rares à l'écrit, et en partie accidentels ${ }^{13}$. En outre, en dehors de quelques cas singuliers comme celui des textes vraiment macaroniques, on peut affirmer que (à l'écrit au moins) chaque situation de rencontre entre les langues se fait sous l'ascendant d'une «langue matrice»-langue principale d'un texte, langue cible d'une traduction - qui se laisse plus ou moins pénétrer par d'autres. C'est ce que rappelle à sa manière Édouard Glissant en signalant que ce que nous appelons «langue matrice» peut exister sur la page selon une plus ou moins grande ouverture:

On peut ne pas parler d'autre langue que la sienne. C'est plutôt la manière même de parler sa propre langue, de la parler fermée ou ouverte; de la parler dans l'ignorance de la présence des autres langues ou dans la prescience que les autres langues existent et qu'elles nous influencent même sans que nous le sachions. Ce n'est pas une question de science, de connaissance des langues, c'est une question d'imaginaire des langues. Ce n'est pas une question de juxtaposition des langues, mais de leur mise en réseau ${ }^{14}$.

En tout état de cause, le multilinguisme se manifeste d'une manière qu'il nous est encore difficile d'embrasser dans l'ensemble de ses possibles. Ầ l'heure où les langues insulaires en contact, et les formes qu'elles prennent dans les manuscrits et les textes qu'ils transmettent, sont au cœur d'une actualité de recherche féconde, il est bon d'interroger à nouveaux frais le mode de présence et d'interaction des langues, d'en dégager les enjeux, pour tenter de comprendre comment les langues peuvent s'inscrire ensemble dans différents champs discursifs.

13. Voici deux exemples de manuscrits que l'on peut qualifier de «quadrilingues»: London, Wellcome Historical Library, ms. 801A, assemblage composite, XII ${ }^{\mathrm{e}}$-XIV ${ }^{\mathrm{e}} \mathrm{s}$. (provenance XIII ${ }^{e}$ s. : abbaye de Bury St Edmunds, Suffolk): latin, anglais, gloses en italien, en anglo-français; et London, British Library (désormais abrégé en: BL), ms. Arundel 292, $\mathrm{XIII}^{\mathrm{e}} \mathrm{s}$. (provenance $\mathrm{XIV}^{\mathrm{e}} \mathrm{s}$. : bibliothèque du chapitre de la cathédrale de Norwich, après l'incendie de 1272): latin, anglo-français, anglais, alphabet hébreu en inscription subsidiaire. Le fait que ces recueils proviennent de deux institutions bénédictines ne semble pas avoir de signification particulière.

14. É. GLISSANT, Introduction à une poétique du Divers, Paris, 1996, p. 122-123. 


\section{Petite histoire des langues d'Angleterre}

Des chronologies pourront d'abord être dégagées en amont de la constitution du trio qui a retenu l'attention de la plupart des contributions. Elles dessinent, selon les espaces-temps des pratiques, selon les langues en présence, cette esthétique de la «ligne brisée» qu'évoque André Crépin au sujet de l'histoire des langues ${ }^{15}$ - ajoutons, de leurs interactions.

Avant l'anglais d'abord. Remonter à la situation linguistique antérieure au Moyen Âge n'a rien d'une évidence, l'historiographie ayant longtemps considéré l'avènement de l'anglais - voire l'adventus des Anglais eux-mêmes - comme une tabula rasa linguistique autant qu'identitaire. Or il n'y a jamais de «monolithisme» à l'intérieur d'une langue dans un espace donné: une langue "parle» toujours d'autres langues en elle, même si la conscience de ce phénomène n'est pas nécessairement le fait de ses locuteurs. Ainsi, contrairement au récit mythifié de la migration anglosaxonne, déjà assumé par les Anglo-Saxons eux-mêmes ${ }^{16}$, il faut affirmer que la population qu'au VIII ${ }^{\mathrm{e}}$ siècle Bède le Vénérable appellera «angle» ne s'est pas substituée de manière brutale et entière à la population des provinces romaines de la Bretagne, mais qu'elle est née de la rencontre entre des populations déjà installées dans l'île - britto-romaines, brittoniques ou pictes - et des groupes nouvellement arrivés - germaniques ou irlandais. La langue anglaise, elle aussi, est née de ces interactions. L'anglais (que l'on appellera «vieil anglais» de préférence au terme «anglo-saxon», qu'on réservera aux phénomènes culturels et historiques non marqués linguistiquement) n'est pas arrivé armé de pied en cap sur les trois navires qui, selon le récit d'origine rapporté dans le De excidio Britanniae de Gildas puis dans la plupart des histoires médiévales de la Bretagne, amenèrent dans l'île les premiers «Saxons ${ }^{17}$ » : il s'est développé au sein d'une matrice insulaire elle-même déjà marquée par le multilinguisme. Les travaux sur les sources lexicographiques du latin insulaire (par exemple les inscriptions du $\mathrm{V}^{\mathrm{e}}-\mathrm{VI}^{\mathrm{e}}$ siècle) montrent la langue latine influencée par le substrat brittonique dans ses réalisations précoces, et surtout existant au Bas-Empire en tant que langue parlée (c'est-à-dire en tant que proto-roman insulaire) dans les lowlands de la moitié sud-est de l'île, là même où dans les siècles suivants se développerait l'anglais ${ }^{18}$; quant au gallois, il porte encore dans sa lexie

15. A. CRÉPIN, Deux mille ans de langue anglaise, Paris, 1994, p. 95.

16. N. Howe, Migration and Mythmaking in Anglo-Saxon England, New Haven/ Londres, 1989.

17. M. COUMERT, Origines des peuples. Les récits du haut Moyen Âge occidental (550850), Paris, 2007, p. 397-400.

18. P. SchriJVer, «The Rise and Fall of British Latin: Evidence from English and Brittonic », dans M. Filppula, J. Klemola et H. PitKänen éd., The Celtic Roots of English, Joensuu, 2002, p. 87-110; A. S. Gratwick, «Latinitas Britannica: Was British Latin 
le témoignage diffracté de ces rencontres complexes avec le latin ${ }^{19}$. Les effets de ces interférences, antérieures aux migrations anglo-saxonnes mais habitant en quelque sorte le territoire où se développerait la langue, se mesurent donc dès l'origine sur l'anglais.

La conscience de la diversité des langues dans l'île autant que dans la chrétienté tout entière est une constante - presque une banalité - de la littérature anglo-saxonne, en vieil anglais ou en latin, depuis Aldhelm à la fin du VII siècle jusqu'à Ælfric autour de l'an mil, en passant par les réflexions du roi Alfred le Grand à la fin du $\mathrm{IX}^{\mathrm{e}}$ siècle ${ }^{20}$. Elle se trouve bien sûr exprimée chez Bède le Vénérable, dans un passage célèbre:

Aujourd'hui celle-ci [l'île de Bretagne], selon le nombre de livres dans lesquels est écrite la Loi divine, étudie et confesse une seule et même connaissance de la plus haute vérité et de la vraie sublimité dans les langues de cinq peuples, à savoir celle des Anglais, celle des Bretons, celle des Irlandais, celle des Pictes et celle des Latins qui par l'étude des Écritures est devenue commune à tous les autres peuples ${ }^{21}$.

La situation qu'évoque Bède correspond à celle de son temps, les premières décennies du VIII ${ }^{\mathrm{e}}$ siècle. Mais si on lit bien ce passage, on constate qu'il ne se situe pas vraiment «au-delà du bilinguisme». Bède identifie plutôt à l'intérieur des îles Britanniques quatre grandes «zones de communication » bien distinctes utilisant chacune leur «langue»-même s'il s'agissait sans doute en réalité d' «aires dialectales» d'intercompréhension plutôt que de régions marquées par une réelle uniformité linguistique - entre lesquelles la «langue des Latins» aurait servi de lingua franca, permettant

Archaic?», dans N. BRooKs éd., Latin and the Vernacular Languages in Early Medieval Britain, Leicester, 1982, p. 1-79.

19. T. M. Charles-EDWARDS, Wales and the Britons 350-1064, Oxford, 2013, p. 112113.

20. Voir en particulier la célèbre préface en prose d'Alfred à sa traduction du Pastoral de Grégoire le Grand, traduite dans A. GAUTIER éd., Asser, Histoire du roi Alfred, Paris, 2013, p. 203-217.

21. BÈDE, Historia ecclesiastica gentis Anglorum, I, 1, 3 (BÈDE LE VÉNÉRABLE, Histoire ecclésiastique du peuple anglais, éd. M. LAPIDGE, A. CRÉPIN, P. Monat et P. RobIN, Paris, 2005, vol. 1 [Sources chrétiennes, 489], p. 114): «Haec in praesenti iuxta numerum librorum quibus lex diuina scripta est, quinque gentium linguis unam eandemque summae ueritatis et uerae sublimitatis scientiam scrutatur et confitetur, Anglorum uidelicet Brettonum Scottorum Pictorum et Latinorum, quae meditatione scripturarum ceteris omnibus est facta communis (notre traduction). » Ce passage doit être rapproché d'un autre, au livre III, 6, 1 (vol. 2 [Sources chrétiennes, 490], p. 42), où Bède explique que les nationes et les prouinciae de la Bretagne usent de quatre langues: celles «des Bretons, des Pictes, des Irlandais et des Anglais». 
en particulier l'accès au savoir religieux ${ }^{22}$. Georges Tugène a montré à quel point cette présentation des cinq langues de la Britannia s'inscrit dans des considérations ecclésiologiques sur les rapports entre gentes, langues et histoire du salut: le christianisme, né de la Pentecôte, est présenté comme l'anti-Babel, il permet l'interaction harmonieuse des langues qui participent toutes, chacune à leur place, à la diffusion de la parole de Dieu mais aussi au chœur eschatologique des langues. Ainsi, l'évocation de la diversité des langues participe chez Bède d' «une véritable "vision" des Églises insulaires qui, parlant toutes les langues de la Britannia, s'unissent dans la louange de Dieu », une vision qui dépasse donc de loin la simple description d'une situation linguistique réelle ${ }^{23}$. Le plurilinguisme (au sens d'une pluralité et d'une coexistence des langues, voire d'une harmonie entre elles) apparaît bien comme «constitutif du christianisme ${ }^{24}$ », mais il est compris comme une diversité linguistique idéale et eschatologique plutôt que comme un véritable multilinguisme (au sens d'une pratique de la diversité des langues vécue par les individus et les groupes).

Dans la pluralité des langues, il faut bien entendu noter la place singulière du latin, que son statut de langue «référentielle», pour reprendre la typologie proposée par Benoît Grévin ${ }^{25}$, met à part de toutes les autres. C'est pour cette raison même que focaliser l'étude sur la coexistence entre le latin et les autres langues nous mènerait à une impasse. Le latin rapproche indéniablement les hommes et les peuples en les faisant communier à un même savoir religieux, il peut même à l'occasion permettre l'interaction entre eux en servant de langue véhiculaire, mais il ne met pas fin à la séparation linguistique qui est au fondement des distinctions ethniques opérées par Bède. Il est vrai que la distance objective qui existe dans le monde insulaire entre le latin, langue référentielle, et les parlers locaux, à l'origine exclus du monde de l'écrit, favorise paradoxalement l'émergence des vernaculaires écrits et la formation d'élites lettrées bilingues: «De la division naît en effet une clarté contrastive. Elle explique au haut Moyen Âge l'avance prise par les langues celtes et germaniques dans l'acquisition d'un registre écrit, par contraste avec le retard des dialectes romans mal discernés du latin ${ }^{26} »$. Mais ce bilinguisme des clercs est-il un authentique multilinguisme? Ne s'apparente-t-il pas plutôt à un «conditionnement,

22. G. TugÈnE, L'Image de la nation anglaise dans l'Histoire ecclésiastique de Bède le Vénérable, Strasbourg, 2001, p. 119-124.

23. ID., L'Idée de nation chez Bède le Vénérable, Paris, 2001, p. 328.

24. Ibid., p. 330.

25. B. GrÉVIn, Le Parchemin des cieux. Essai sur le Moyen Âge du langage, Paris, 2012, p. 18: sont appelés référentiels «les langages que leur rôle de supports de corpus textuels fondateurs de leurs idéologies sacralisaient dans ces sociétés, en faisant d'eux les références ultimes de la pensée et de la pratique linguistique».

26. Ibid., p. 98. 
d'ordre formel et didactique, [qui] s'accomplit au cours d'une éducation qui façonne le lettré pour le mettre en possession de ces vecteurs de la science et de la foi que sont les langues référentielles ${ }^{27} \gg$ ? Une fois ce conditionnement pris en compte - et il agit puissamment sur la manière dont les langues vulgaires sont réalisées à l'écrit - le face à face entre le latin et le vernaculaire, fût-il écrit, semble bien laisser le lettré prisonnier de Babel: Bède ne connaît ni l'irlandais, ni le gallois. À rebours de Bède, c'est donc bien la Babel des situations d'interaction du présent des acteurs qui retiendra notre attention, et non la Pentecôte eschatologique qu' annonce si mal la diffusion universelle du latin. D'où le choix de notre illustration de couverture: une représentation de l'épisode de la tour de Babel tirée du manuscrit généralement connu sous le nom d' «Hexateuque vieil anglais ${ }^{28} »$.

Aussi de nombreux spécialistes ont-ils eu tendance à étudier les situations linguistiques des différentes gentes des îles Britanniques dans le haut Moyen Âge, non pas comme des situations de trilinguisme, mais avant tout comme des bilinguismes latin-vernaculaire juxtaposés ${ }^{29}$. Pourtant la lecture des sources, à commencer par Bède lui-même, nous montre que le latin n'assurait pas seul l'intercompréhension entre des univers linguistiques étanches. Lors de l'atelier préparatoire à la présente publication, Olivier Szerwiniack a recensé et présenté les situations d'interaction entre langues vernaculaires, qui s'avèrent en fin de compte assez nombreuses dans l'œuvre de Bède: les contacts entre anglais et irlandais (directs ou par l'intermédiaire du latin) sont fréquents, ceux entre anglais et picte le sont un peu moins, et ceux entre anglais et breton sont relégués par Bède à quelques rares instances - non pas parce qu'elles auraient été moins nombreuses, mais parce qu'il entre dans le projet politico-religieux de l'auteur de reléguer les Bretons et d'insister sur leur isolement d'avec les autres chrétiens, signe et conséquence de leur endurcissement ${ }^{30}$. Et cela sans parler d'autres langues qui apparaissent au détour de l'œuvre de Bède: le grec dont l'étude s'impose à l'école cathédrale de Cantorbéry sous la houlette de l'archevêque Théodore de Tarse; l'hébreu dont Bède s'essaie à commenter quelques termes dans ses œuvres exégétiques; ou encore le «parler barbare» (francique germanique ou roman de la région parisienne?)

27. Ibid., p. 189.

28. Le ms. London, BL, Cotton Claudius B.iv est un ms. richement illustré, sans doute copié à l'abbaye Saint-Angustin de Cantorbéry dans la première moitié du XI siècle. Il contient la traduction en vieil anglais par Ælfric d'Eynsham des six premiers livres de l'Ancien Testament, à savoir le Pentateuque et Josué.

29. A. ORCHARD, «Latin and the Vernacular Languages: The Creation of a Bilingual Culture», dans T. Charles-Edwards éd., After Rome, Oxford, 2003, p. 190-219.

30. O. SzERWINIACK, «Les langues dans les îles Britanniques selon l'Histoire ecclésiastique de Bède», communication présentée à l'atelier «Les langues de l'Angleterre médiévale: au-delà du bilinguisme », Poitiers, 13 septembre 2013. 
de l'évêque franc Agilbert, qui irritait le roi ouest-saxon Cenwealh (643674) parce qu'il ne connaissait lui-même «que la langue des Saxons », c'està-dire la forme d'anglais parlée dans le sud de l'île ${ }^{31}$. Un comble quand on sait que, cinq siècles plus tard, les nobles anglais enverront leurs enfants en France afin d'éradiquer «le caractère barbare de leur langue natale ${ }^{32} »$ !

Ce plurilinguisme est demeuré plus ou moins stable jusqu'aux invasions scandinaves, dont les péripéties dominent à maints égards l'histoire politique de l'Angleterre pendant plus de deux siècles et demi, des premiers raids vikings des années 780 jusqu'au dernières menaces norvégiennes et danoises des années 1060-1080. L'irruption puis l'implantation durable du norrois marquent alors l'Angleterre autant que d'autres régions insulaires, dont certaines (comme les îles Shetland, Orcades et Hébrides) voient leur situation linguistique profondément transformée. En Angleterre même, l'adstrat scandinave intervient donc sur un substrat déjà au moins bilingue, voire dès l'origine multilingue. Son développement complique sur la longue durée le jeu sur les formes de contact, ce qui se manifeste de deux manières principales. D'une part, la proximité linguistique entre le norrois et le vieil anglais facilite l'ouverture de la langue indigène à l'influence de la langue importée en multipliant les occasions de contact direct entre les individus. On a discuté de l'intercompréhension entre les locuteurs des deux langues, mais quelle qu'ait été sa mesure, il ne fait pas de doute que les contacts directs étaient plus aisés entre locuteurs de ces deux langues germaniques qu'avec les locuteurs de langues romanes et celtiques ${ }^{33}$. D'autre part, l'influence du norrois ne s'impose pas de manière uniforme (géographique ou chronologique) dans l'Angleterre des $\mathrm{IX}^{\mathrm{e}}-\mathrm{X}^{\mathrm{e}}$ siècles. Les parlers scandinaves impriment plus fortement leur marque sur les régions du Nord et de l'Est de l'île, où la domination viking et l'implantation de colons scandinaves sont beaucoup plus prégnantes. Arnaud Lestremau, reprenant une formulation remontant à Marc Bloch, a ainsi montré par des analyses chiffrées que le vieux norrois a bien représenté un «apport en profondeur» dans l'anthroponymie du monde anglo-saxon au cours du siècle précédant la Conquête normande; il a surtout montré que cette présence des noms norrois valait pour toute l'Angleterre, mais selon un gradient nord-est/sud-ouest très sensible ${ }^{34}$. Par ailleurs, le norrois s'est imposé de manière différenciée selon les époques, les vagues d'implantation et les catégories sociales: l'immigration scandinave numériquement plus

31. BÈDE, Historia ecclesiastica gentis Anglorum, III, 7, 4 (éd. citée, vol. 2, p. 48): «barbara loquella».

32. Voir ci-dessous l'article de C. Lucken.

33. M. Townend, Language and History in Viking Age England: Linguistic Relations between Speakers of Old Norse and Old English, Turnhout, 2002.

34. A. Lestremau, Pratiques anthroponymiques et identités sociales en Angleterre (mi$X^{e}$-mi-XI siècles), thèse de doctorat inédite, Université Paris I, 2013, p. 606-614. 
importante du troisième tiers du IX ${ }^{\mathrm{e}}$ siècle, mais pour l'essentiel confinée aux régions du Danelaw et marquée par une implantation dans les terroirs et une appropriation des terres agricoles, n'a pas pu avoir les mêmes effets linguistiques que l'apport bien moins nombreux, géographiquement mieux réparti mais limité à une étroite élite administrative et militaire, qui a fait suite à la conquête du royaume par le Danois Cnut le Grand (1016-1035). La culture anglo-saxonne fut donc dès le début éminemment multilingue, et ce multilinguisme ne cessa de s'enrichir au fil des siècles séparant la fin de l'Antiquité de la Conquête normande. Malgré cela, le paradigme du bilinguisme demeure très prégnant dans son historiographie, qu'il s'agisse du bilinguisme anglais-norrois ${ }^{35}$ ou du bilinguisme anglais-latin, exploré de manière particulièrement fructueuse dans la lexie de l'anglais ${ }^{36}$.

Une autre «complication» dans la chronologie des langues d'Angleterre est évidemment celle qui résulte des effets de la Conquête normande. Car l'intégration de l'Angleterre à l'ensemble anglo-normand n'a pas eu pour seul effet de faire advenir le français en Angleterre, ou le français d'Angleterre: le multilinguisme du pays s'est renouvelé à ce creuset de multiples façons. Ainsi le latin, et pas seulement le français, a-t-il progressé dans l'usage administratif dont l'anglais, jusque-là très présent, s'est retrouvé très largement exclu ${ }^{37}$. Ce renouvellement a d'ailleurs commencé dès avant la Conquête, comme le rappelle Elaine Treharne ${ }^{38}$. Le $\mathrm{XII}^{\mathrm{e}}$ siècle, temps d'absorption de la Conquête, apparaît alors comme une période d'expérimentations et de perméabilité des cultures: pour citer Ian Short, «ce qui donne au contexte littéraire de l'Angleterre du XII ${ }^{\mathrm{e}}$ siècle son caractère unique, c'est la confluence de trois processus sociaux : renouveau intellectuel, symbiose culturelle et vernacularisation du savoir ${ }^{39} »$. Cette vernacularisation ne signifie pas pour autant un recul ou un déclin du latin: même si l'Angleterre se retrouve désormais annexée à l'aire de culture romane, elle ne diffère pas nécessairement du reste de l'Europe du

35. En témoignent par exemple les travaux de M. TownEnd, «Viking Age England as a Bilingual Society», dans D. M. HADLEY et J. D. Richards éd., Cultures in Contact: Scandinavian Settlement in England in the Ninth and Tenth Centuries, Turnhout, 2000, p. 89105 ; ID., Language and History...

36. H. GNEUSS, «Anglicae linguae interpretatio: Language Contact, Lexical Borrowing and Glossing in Anglo-Saxon England», Proceedings of the British Academy, 82 (1993), p. 107-148.

37. M. Clanchy, From Memory to Written Record: England 1066-1307, $3^{\mathrm{e}}$ éd., Oxford, 2013, p. 24-25.

38. E. M. Treharne, Living through Conquest. The Politics of Early English, 10201220, Oxford, 2012.

39. I. ShORT, «Language and Literature», dans C. HARPER-Bill et E. VAn Houts éd., A Companion to the Anglo-Norman World, Woodbridge, 2007, p. 191-214 (p. 210). 
Nord où le latin reste au XII ${ }^{\mathrm{e}}$ siècle une langue «conquérante ${ }^{40}$. Dans sa transmission manuscrite, le vieil anglais se «réécrit» alors à l'horizon des langues nouvellement en présence ${ }^{41}$. Ainsi le célèbre manuscrit Ii.3.33 de la Cambridge University Library, copié à Ely à la fin du XII ${ }^{\mathrm{e}}$ siècle, et qui contient une sélection de textes en vieil anglais de l'auteur anglo-saxon Ælfric d'Eynsham (sermons, vies de saints, une partie de son Hexateuque), constitue la plus importante collection attribuable à un auteur en langue anglaise avant Chaucer. Pourtant, comme l'a montré Elaine Treharne, si les textes copiés sont bien écrits dans la norme ouest-saxonne en vigueur dans l'Angleterre d'avant la Conquête normande, une étude plus fine du manuscrit révèle des dynamiques plus subtiles, propres au milieu multilingue d'un monastère anglais de l'époque angevine ${ }^{42}$.

Finissons ce rapide parcours chronologique avec les trois derniers siècles du Moyen Âge, sur lesquels se concentrent la plupart des contributions finalement publiées dans ce numéro. Les XIII et XIV siècles s'avèrent déterminants pour le développement de la compilatio et de l'ordinatio, selon les termes retenus par Malcolm B. Parkes: le passage d'un mode de lecture linéaire propre aux contextes monastiques à des pratiques plus dynamiques propres au monde scholastique entraîne des changements dans la structuration des livres, avec la multiplication des rubriques, sauts de lignes, titres de chapitres, gloses marginales, sommaires et index ${ }^{43}$. De tels développements sont éminemment propices à la coexistence des langues sur la page manuscrite, puis imprimée. Dans le même temps, les aléas politiques et militaires - avec la perte, la conquête puis à nouveau la perte de vastes territoires dans le royaume de France - ont des effets sensibles sur les rapports entre les trois principales langues. L'anglais ayant fermement fait retour au monde de l'écrit, la hiérarchisation des trois langues, les choix qui sont faits de les utiliser ou de les combiner selon les milieux ou le type d'information véhiculée connaissent de nombreux changements et une grande diversité de situations dans ces trois siècles, qui apparaissent en Angleterre comme un âge d'or du trilinguisme ${ }^{44}$. Pour ne citer qu'un seul exemple, le français d'Angleterre continue à développer

40. L. B. Mortensen, «Roman Past and Roman Language in Twelfth-Century English Historiography », dans E. M. TYLER, Conceptualizing Multilingualism..., p. 309-320 (p. 312).

41. M. Swan et E. M. Treharne éd., Rewriting Old English in the Twelfth Century, Cambridge, 2006.

42. E. M. Treharne, «The Vernaculars of Medieval England, 1170-1350», dans A. Galloway éd., The Cambridge Companion to Medieval English Culture, Cambridge, 2011, p. 217-236.

43. M. B. PARKES, «The Influence of the Concepts of Ordinatio and Compilatio on the Development of the Book», dans J. J. G. AleXANDER et M. T. GiBson éd., Medieval Learning and Literature. Essays Presented to Richard William Hunt, Oxford, 1976, p. 115-141.

44. Sur la hiérarchisation des langues et la répartition des domaines et des registres d'usage des langues au Moyen Âge, voir en dernier lieu la synthèse, chronologiquement et 
des caractères propres, ceux d'un «latin bis» qui, par l'intermédiaire de la langue de chancellerie de la royauté anglaise, donnera plus tard naissance au Law French (qui a subsisté dans la pratique juridique jusqu'en plein $\mathrm{XVIII}^{\mathrm{e}}$ siècle), tout en influençant profondément la graphie et le lexique du moyen anglais ${ }^{45}$.

On pourrait alors faire l' hypothèse de deux formes de multilinguisme : pour une part, ce qu' on pourrait appeler un «multilinguisme de contact», où ce qui est en jeu, c'est l'entrée brute, dans une langue donnée, d'un lexème, d'une tournure, d'une valeur de sens venus d'une autre langue; pour une autre, ce qu'on dirait un «multilinguisme culturel», où ce qui importe, c'est le consentement à l'appropriation ou à l'embrassement de réalités linguistiques initialement distinctes, à l'entrée dans ce que Serge Lusignan appelle joliment la «ronde des échanges linguistiques ${ }^{46}{ }$, mais aussi dans celle des relations et des symbioses profondes. Toute l'affaire du multilinguisme anglais médiéval, c'est qu'il oscille toujours entre ces deux tendances, esquissant les formes d'une sociolinguistique historique singulière.

\section{Quand les langues entrent en contact}

La pratique des langues n'est jamais désincarnée. Elle est affaire d'institutions, de groupes socio-professionnels (pensons aux langues du droit étudiées par Paul Brand ${ }^{47}$ ), d'individus, affaire de génération et d'usage, d'habitus dominant et de métamorphoses des pratiques ${ }^{48}$, affaire de médiatisation par l'écrit, documentaire ou littéraire, collectif ou privé. Elle est aussi le reflet décalé de l'histoire politique, de son reflux sur les pratiques autant que sur les individus. Car le rapport entre les langues est autant un ensemble de faits qu'une idéologie, avec de nouvelles déclinaisons en notre temps. Le modèle de tripartition fonctionnelle quasidumézilienne entre le latin (langue de l'Église, de l'auctoritas et de la fonction de souveraineté), l'anglo-normand (langue de la cour, de la chevalerie, de la domination seigneuriale et de la fonction guerrière), et

géographiquement bien plus ambitieuse que le présent dossier, proposée par B. GRÉVIN, $L e$ Parchemin des cieux...

45. S. Lusignan, La Langue des rois au Moyen Âge. Le français en France et en Angleterre, Paris, 2004.

46. ID., «Le français et le latin aux XIII ${ }^{\mathrm{e}}-\mathrm{XIV}^{\mathrm{e}}$ siècles: pratique des langues et pensée linguistique», Annales E.S.C., 42/4 (1987), p. 955-967 (p. 961).

47. P. BRAND, «The Language of the English Legal Profession: The Emergence of a Distinctive Legal Lexicon in Insular French», dans R. InGHAM éd., The Anglo-Norman Language and its Contexts, York, 2010, p. 94-101.

48. Issue, on le sait, des travaux de Pierre Bourdieu, la notion d'habitus a gagné en audience; sans doute est-elle utilisée en excès dans C. BREEN, Imagining an English Reading Public, Cambridge, 2010. 
l'anglais (langue de la quotidienneté, du travail, de la sphère domestique et de la fonction de production) s'est essoufflé, tout comme s'est essoufflé le master narrative du «triomphe de l'anglais ${ }^{49} »$. Diverses tentatives de «redynamisation» ont été faites, par exemple par Tim Machan, qui affirme qu' «en termes très généraux [...], il est bien possible que le latin ait été la langue de l'autorité, le français celle de la cour et du droit, l'anglais celle de l'expérience quotidienne. Toutefois, à l'intérieur de ces termes généraux, il restait toujours possible d'exploiter les subtilités et les nuances qu'offrait le contact des langues dans l'Angleterre médiévale ${ }^{50}$ ».

Il n'en reste pas moins que la pluralité des langues, mais aussi la diversité de leurs usages et surtout de leurs interactions, apparaissent irréductibles à ces schémas. Ici la réflexion doit se faire phénoménologique: il s'agit de se pencher sur les rapports entre livre et corps (ou chair), livre et intentionnalité, livre et langue, et de mettre en avant une nouvelle conception de la «vernacularité ${ }^{51} »$. Avant même de discuter les fonctions des trois langues principales, il convient de remarquer que les autres langues présentes dans l'espace anglais au Moyen Âge ne se laissent pas réduire pas même de façon grossière - à l'une des trois fonctions canoniques. Ainsi de l'hébreu, qui participe tout autant de chacune d'entre elles. De fait, en tant que vernaculaire oral, l'hébreu a une durée de vie contingentée par les effets de l'histoire politique, avec l'expulsion des juifs du royaume en $1290^{52}$ : on se référera ici aux apports de la sociolinguistique historique mis en lumière dans le travail de Bernard Spolsky ${ }^{53}$. Mais il est bien évident qu'en tant que langue écrite, les traces de l'hébreu sont présentes bien avant la Conquête et demeurent bien au-delà de l'expulsion de 1290. Le mérite revient à David Trotter, dans «Peut-on parler de judéo-anglo-normand? Textes anglo-normands en écriture hébraïque», d'avoir conjugué la question de l'anglo-normand en contact avec l'hébreu (mais aussi, dans une moindre mesure, le latin) à celle d'une interrogation sur sa capacité «idiolectale» à

49. Comme le remarque A. MAIREY, «Multilinguisme et code-switching en Angleterre à la fin du Moyen Âge. Approche historiographique», Cahiers électroniques d'histoire textuelle du LaMOP , 2 (2009), p. 29-68 (en ligne: http://lamop.univ-paris1 fr/spip.php?rubrique218).

50. T. W. Machan, «Medieval Multilingualism and Gower's Literary Practice», Studies in Philology 103/1 (2006), p. 1-25 (p. 11-12): «In very general terms [...], Latin may well have been the language of authority, French of the court and law, and English of daily experience. Within these general terms, however, were opportunities for the exploitation of the subtleties and nuances of language contact in medieval England. »

51. M. WORLEY, «Using the Ormulum to Redefine Vernacularity», dans F. SOMERSET et N. Watson éd., The Vulgar Tongue. Medieval and Postmedieval Vernacularity, University Park, 2003, p. 19-30.

52. E. De Visscher, «Hebrew, Latin, French, English: Multilingualism in JewishChristian Encounters», dans J. JeFFERSon et A. PutTer éd., Multilingualism in Medieval Britain (ca 1066-1520). Sources and Analysis, Turnhout, 2013, p. 89-103.

53. B. SpOlsky, The Languages of the Jews. A Sociolinguistic History, Cambridge, 2014. 
incarner, à travers certaines gloses, une sorte de nouvel idiome, le judéoanglo-normand - comme on parle de judéo-français. Cette spécificité se marque par exemple à travers le fait qu'un mot «anglo-normand» transcrit en alphabet hébreu peut s'avérer être d'origine anglo-saxonne, et propre au lexique du français d'Angleterre ${ }^{54}$.

Bien entendu, en Angleterre autant qu'ailleurs et depuis plus longtemps, la question de la compétence grammaticale et rhétorique, et plus largement celle de la literacy, n'était l'apanage d'aucune langue en particulier, et surtout pas de la seule expression en latin. Les sources écrites en témoignent largement: livres liturgiques, sources documentaires, recueils de textes de domaines divers, de la théologie à la Fachliteratur. Mais le prisme du fait manuscrit peut imposer une distorsion : l'«oraliture» - néologisme proposé par Patrick Chamoiseau pour penser les effets d'une littérature produite à partir de l'oralité - supporte assez mal son passage en «littérature ${ }^{55}$. On mentionnera, entre autres textes emblématiques, l'ouvrage exégétique intitulé Orrmulum (XII ${ }^{\mathrm{e}}$ siècle) ${ }^{56}$, les sermons macaroniques du XVe $\mathrm{XVI}^{\mathrm{e}}$ siècle étudiés par Alan Fletcher ${ }^{57}$ - un genre moindrement trilingue cependant - ou encore les annotations et traductions d'hymnes et d'antiennes du franciscain William Herebert de Hereford au début du XIV ${ }^{\mathrm{e}}$ siècle $^{58}$. Le latin, langue référentielle, reste «instrument de conceptualisation et guide d'écriture ${ }^{59}{ }$ et continue d'exercer une pression sur l'écrit vernaculaire.

Malgré ces avancées récentes, on manque encore d'un véritable cadre de réflexion pour la transmission écrite de plusieurs langues ensemble. On relève certes de plus en plus de manuscrits trilingues et, au-delà du simple relevé bibliographique, ces manuscrits comptent de plus en plus dans la conscience critique. Mais comment penser, par exemple, le «trilinguisme discret», limité à un point d'un manuscrit ? Dans la communication qu'il a présentée lors de l'atelier préparatoire ${ }^{60}$, Jean-Pascal Pouzet s'est attaché à

54. Voir ci-dessous le commentaire de David Trotter sur le mot malve translittéré en hébreu dans le ms. Valmadonna 1.

55. P. Chamoiseau, Texaco, Paris, 1992; et ID., Écrire en pays dominé, Paris, 1997.

56. Voir à ce sujet J.-P. PouZET, «Voix, écriture et lyrisme dans l'Orrmulum», communication présentée au colloque «Voix saintes» du Centre d'études médiévales anglaises de l'Université Paris IV, 22-23 mars 2013.

57. A. J. FletChER, The Grammar of Good Friday. Macaronic Sermons of Late Medieval England, Turnhout, 2012.

58. London, BL, ms. Add. 46919, fo 205 $\mathrm{r}^{\mathrm{o}}$ : colophon latin de William Herebert, traducteur et scribe. Voir H. GNEUSS, «William Herberts Übersetzungen », Anglia. Zeitschrift für englische Philologie, 78 (1960), p. 169-192 (p. 174).

59. B. GRÉVIN, Le Parchemin des cieux..., p. 306-307.

60. J.-P. PouzET, «Multilinguisme et codicologie du XIII ${ }^{\mathrm{e}}$ au $\mathrm{XV}^{\mathrm{e}}$ siècle: nouvelles approches », communication présentée à l'atelier «Les langues de l'Angleterre médiévale: au-delà du bilinguisme», Poitiers, 13 septembre 2013. 
définir, à partir d'exemple précis, les périmètres réglant l'interaction entre forme physique et contenu linguistique. Il a ainsi exploré des corrélations entre des fonctionnements de langues et de formes matérielles dans l'Angleterre du XIII' au XV siècle, ces quelque trois siècles de l'apogée du trilinguisme à l'écrit. Des concepts nouveaux ont été proposés, comme celui de «chaîne stratigraphique», qui signe autant une relation entre des constituants du codex qu'une relation entre des événements: la mise en contact physique entre les langues relève d'une grande complexité «procédurale». Ainsi un recueil multilingue, dans lequel coexistent des textes dont les langues matrices sont différentes, ne résulte pas toujours de la réunion a posteriori d'unités codicologiques hétérogènes, car la contiguïté des langues matrices peut résulter d'un projet du concepteur du recueil: dans le cas d'un recueil homogène, cette contiguïté entre des sous-unités linguistiquement diverses peut être marquée par des éléments de discontinuité, mais elle peut aussi présenter des éléments soulignant la continuité et l'unité du projet codicologique de part et d'autre de la frontière entre les deux sous-unités.

Alors comme aujourd'hui, les langues ont les valeurs qu'on leur prête; leurs interactions aussi. Dès le XII ${ }^{\mathrm{e}}$ siècle, l'assurance nouvelle du français en tant que langue normée a peut-être été acquise non seulement à l'imitation du latin, mais aussi au contact de l'anglais qui, dans le dernier siècle de l'histoire anglo-saxonne, avait lui-même été une langue normée (sinon dotée de véritables traités de grammaire) et bénéficiant d'un statut élevé: longtemps tenu «en dehors de l'emprise de la grammaire ${ }^{61} \gg$ comme la plupart des idiomes «naturels», le français aurait alors bénéficié de «the confidence of English as a written vernacular ${ }^{62} \gg$. Mais de quel français parlet-on? Dans «Le beau français d'Angleterre. Altérité de l'anglo-normand et invention du bon usage », Christopher Lucken montre comment le jugement des contemporains sur la qualité du français (des français) parlé(s) - «faus franceis» d'Angleterre, «doulz françois ou romance» réputé de France - est en grande partie informé par la plus grande normativité du «latin d'école». Ici la pluralité s'invite au cœur des langues en même temps que dans leur hiérarchie: dans les ouvrages normatifs ici étudiés, le français «de France» est présenté comme plus pur et mieux prononcé que l'anglo-normand; pourtant, parce qu'elle est prononcée sur l'île de manière uniforme, le chroniqueur Ranulf Higden juge la langue des Normands supérieure à l'anglais, dont la grande diversité dialectale lui apparaît comme un signe de corruption ${ }^{63}$.

61. B. GRÉvin, Le Parchemin des cieux..., p. 179.

62. E. M. TYLER, «England and Multilingualism...», p. 12.

63. Voir ci-dessous le commentaire par C. Lucken du Polychronicon Ranulphi Higden Monachi Cestrensis, together with the English Translations of John Trevisa and of an 
Dans «John Gower ou le multilinguisme en action», Aude Mairey reprend le dossier de l'«attachement au multilinguisme» d'un poète contemporain de Geoffrey Chaucer. Elle signe l'une des rares présentations générales en français de cette œuvre et des interactions qui ne manquent pas de s'établir entre ses trois branches poétiques, qui sont comme trois veines complémentaires et entrelacées. Cet entrelacement déjoue la tentation d'enfermer chaque langue dans une fonction déterminée, sans ouverture vers les autres langues ${ }^{64}$. De fait, l'une des tendances récentes les plus importantes est de réinscrire l'œuvre du poète dans sa dimension trilingue ${ }^{65}$, un point qu'Aude Mairey développe en suggérant que l'œuvre de Gower témoigne de phénomènes de translatio «externe» (par exemple à travers la compilation d'auteurs anciens) autant que de translatio «interne», entre poèmes composés ou non dans des langues matrices différentes. Cette idée de translatio «interne» pourrait être étendue à d'autres aspects de l'œuvre de Gower, comme par exemple la métrique: le «décasyllabe accentué» marque ses Cinkante Balades autant que son Traitié selonc les auctours pour essampler les amantz marietz ${ }^{66}$.

De la translatio on passe tout naturellement à la traduction, situation où une langue est en quelque sorte invitée dans l'intimité d'une autre. Lors de l'atelier préparatoire ${ }^{67}$, Emmanuelle Roux a présenté le cas des traductions de la Somme le Roi, manuel d'instruction religieuse pour les laïcs écrit pour le roi de France par le frère Laurent d'Orléans en 1279. Il existe cinq traductions partielles en moyen anglais de cette œuvre, datant des XIV et $\mathrm{XV}^{\mathrm{e}}$ siècles. Deux d'entre elles contiennent des extraits en latin, souvent réduits à une pure fonction d'autorité; quant au français, les traducteurs le connaissent assez bien, malgré la présence d'erreurs ou de maladresses dans les traductions. Leur anglais apparaît surtout constellé d'expressions françaises, ce qui s'explique en partie si l'on choisit de voir dans l'anglais une langue «en construction», lacunaire dans certains domaines et donc ouverte aux emprunts et néologismes. Pourtant cette explication ne suffit pas: l'existence de doublets lexicaux montre bien que les prétendues lacunes de la langue n'épuisent pas la question. L'auteure s'interrogeait alors sur la notion même de multilinguisme chez les traducteurs, dont elle restituait l'activité

Unknown Writer of the Fifteenth Century, éd. C. B ABIngton, J. R. LumBy, Londres, 1869-86, t. II, p. 156-163.

64. A. MAIREY, «Multilinguisme et code-switching...».

65. E. DutTon et al. éd., John Gower, Trilingual Poet: Language, Translation and Tradition, Cambridge, 2010.

66. M. J. Duffell et D. BiLly, «Le décasyllabe de John Gower ou le dernier mètre anglo-normand», Revue de linguistique romane, 69 (2005), p. 73-95.

67. E. Roux, «Du multilinguisme ou de l'interaction plurilingue? Sur quelques traductions anglaises de La Somme le Roi au XV siècle», communication présentée à l'atelier «Les langues de l'Angleterre médiévale: au-delà du bilinguisme», Poitiers, 13 septembre 2013. 
contribuant à l'enrichissement et à la formation de la langue anglaise. Par «multilinguisme», il ne faudrait donc pas entendre, à la fin du Moyen Âge, le fait «de parler concurremment deux ou trois langues différentes mais d'en utiliser une, d'en faire interagir d'autres, de les manipuler dans le seul but d'aider l'une et l'autre à se construire, se normaliser, s'émanciper ${ }^{68}{ }^{\prime}$. C'est là une belle idée : les langues s'entraident dans leur accomplissement mutuel. C'est le cas pour la résolution philologique des obscurités, quand la scripta anglaise se refuse à trahir la connaissance mêlée mais finalement sûre qu'elle a des autres langues, le français avant tout mais aussi le latin. La traduction n'est donc pas d'abord le lieu privilégié du multilinguisme, mais celui où l'interaction entre les langues permet à l'une d'entre elles - ici l'anglais - de gagner en assurance, à travers un jeu subtil de néologismes, de gallicismes et de calques linguistiques.

Décidément, même ceux qui voudraient toujours reconnaître une pertinence au paradigme du «triomphe de l'anglais» seront forcés d'admettre que cette marche triomphale ne procède pas d'une exclusion des autres langues, mais bien d'une renégociation des relations entretenues avec elles. Car l'intimité entre les langues ne laisse pas indemnes les textes où elle se déroule: la langue matrice - langue cible d'une traduction ou langue principale d'une composition originale - est singulièrement affectée par ces va-et-vient et assume ce qu'elle puise aux langues qu'en même temps elle s'efforce de mettre de côté, voire d'effacer. L'emprunt est une facette de cette intimité, le «saut de langue» (code-switching) en est une autre: si l'on a raison de distinguer entre les deux phénomènes, on ne saurait pour autant ériger une démarcation trop claire. Dans leur essai «Le déclin du multilinguisme dans The Boke of Noblesse et son codicille de William Worcester», Daniel Wakelin et Catherine Nall posent la question d'un éventuel déclin du multilinguisme dans la pratique individuelle d'un literatus de la seconde moitié du $\mathrm{XV}^{\mathrm{e}}$ siècle. Le multilinguisme demeure certes un trait dominant de la culture anglaise de ces années, et un habitus indispensable pour quiconque est conduit à consulter des archives et documents en diverses langues (latin et français, mais aussi à l'occasion gallois ou cornique); pourtant l'analyse de Daniel Wakelin et Catherine Nall tend à montrer que cet habitus devient réticent chez William Worcester (1415-1483), et ce en dépit (peut-être même à travers) la fréquence du codeswitching dans l'œuvre.

\section{Poétiques d'une créolité}

Ces lignes ont à plusieurs reprises invoqué le nom du poète martiniquais Édouard Glissant: il est vrai que l'Angleterre de la Conquête 
a pu être présentée comme une terre marquée par une domination de type colonial où se développent des phénomènes de créolisation. Cette Angleterre du second Moyen Âge, où les rapports entre les langues sont en permanence renégociés dans chacune des situations d'interaction qui ne cessent de se présenter, ne relèverait-elle pas du même champ de tensions que celui qu'Édouard Glissant, dans le Discours antillais, repère entre une «poétique libre, ou naturelle» et une «poétique forcée», forme de résilience dans une créativité contrainte ${ }^{69}$ ? Pour le poète, la "poétique forcée » procède d'une tension entre une nécessité d'expression scribale (le poème doit être transmis) et les formes par lesquelles cette expression est forcée de passer: une «confrontation se noue dans une opposition entre le contenu exprimable et la langue suggérée ou imposée». La «poétique libre» est «tension collective vers une expression» «qui ne s'oppose à ellemême ni au niveau de ce qu'elle veut exprimer ni au niveau du langage qu'elle met en œuvre», le langage des formes scribales incarnant alors celui des formes linguistiques, de leur rencontre.

Pourrait-on alors (même difficilement, même si analogie n'est pas identité) cerner un dilemme, un drame culturel de cet ordre dans les siècles qui ont suivi la Conquête, entre une «poétique naturelle» et une «poétique forcée»? D'une part une coexistence ancienne, peut-être en un premier temps contrainte mais devenue au fil des siècles si «naturelle», entre la langue référentielle qu'est le latin et la langue de tous qu'est l'anglais; d'autre part l'adjonction du français et le développement de l'anglo-français, greffe politique sous contrainte ${ }^{70}$. Car le pouvoir normand a lui-même usé et abusé des procédures multilingues, auxquelles il a contraint le pays tout en étant lui-même forcé d'y recourir afin de pouvoir le gouverner, comme le montrent de nombreux travaux récents sur le Domesday Book et l'enquête qui a précédé sa compilation, «a multilingual and vociferously oral process », pour reprendre les termes de Michael Clanchy ${ }^{71}$.

Ce drame, qui connaîtrait peut-être son apogée à l'époque angevine, aurait alors trouvé une résolution progressive à partir du XIII ${ }^{\mathrm{e}}$ siècle. Entre autres évolutions, cette résolution pourrait avoir été facilitée à partir de 1215 par les grandes entreprises pastorales et pénitentielles, lancées en concile et relayées avec une acuité particulière en Angleterre: la prolifération après Latran IV de l'activité de prédication encourage au moins le bilinguisme, et même souvent un trilinguisme, qui n'est pas forcément tourmenté. Songeons aux découvertes de Siegfried Wenzel, qui a détecté dans certains manuscrits des fragments enchâssés en anglais et en français là où le monolinguisme

69. É. Glissant, Discours antillais, Paris, 1981 (rééd. «Folios Essais», 1997), p. 401.

70. Voir sur ce point E. M. TREHARnE, «The Vernaculars...».

71. M. Clanchy, From Memory...,p. 37. Voir par exemple la belle étude de S. BAXTER, «The Making of Domesday Book and the Languages of Lordship in Conquered England», dans E. M. TYLER éd., Conceptualizing Multilingualism..., p. 271-308. 
latin était jusqu'alors attendu ${ }^{72}$. Ainsi, au cours des siècles qui vont de la Conquête normande au temps des désillusions consécutives à l'échec anglais dans la guerre de Cent Ans, une «poétique libre», ouverte sur d'autres langues, aurait progressivement pris l'ascendant sur les contraintes des siècles précédents, sans renoncer pour autant à faire de la langue - des langues - un champ d'affrontements. On nous permettra donc de conclure en citant Mark Amsler, dont le propos articule plusieurs des thèmes que notre introduction a tenté de présenter:

Dans l'Angleterre du $\mathrm{XIV}^{\mathrm{e}}-\mathrm{XV}^{\mathrm{e}}$ siècle, les interactions multilingues entre anglais, anglo-normand, latin et diverses langues hybrides sont la norme plutôt que l'exception: cela est vrai pour les marchands échangeant leurs biens, pour les diplomates transportant des informations, pour les prédicateurs, pour les travailleurs et les intendants des domaines ruraux, pour les agents et les alliés de Richard II ou d'Henri IV, pour les poètes de cour ou les compilateurs de collections de recettes médicales. Bien souvent, les gens parlaient, entendaient, écrivaient, lisaient à travers les langues: cela vaut pour la communication et pour la parodie, pour l'expression et pour la résistance. L'anglais, l'anglo-normand et le latin étaient alors des langues vivantes. Elles étaient souvent combinées au niveau de la phrase ou du lexique, mais distribuées de façons différentes, pourvues de valeurs différentes, de manière inégale, à l'intérieur d'une même communauté de langage. Car seul un surcroît d'écriture, un surcroît de jeu, un surcroît de contre-commentaire peut résister à l'action d'une idéologie de la langue d'autorité. Dans l'espace de la performance comme sur la page du manuscrit, le latin créolisé révèle que l'autorité linguistique est tout sauf naturelle. $\mathrm{Au}$ contraire, les textualités contestées et les nouveaux hétérotopes sont renforcés par la langue créole, par les énoncés déterritorialisés, par les sujets à la literacy hybride, par les imaginaires multilingues ${ }^{73}$.

72. S. Wenzel, «French Proverbs from the Mouths of English Preachers?», dans C. Bel, P. Dumont et F. Willaert éd., 'Contez me tout': mélanges de langue et de littérature médiévales offerts à Herman Braet, Louvain, 2010, p. 543-555.

73. M. AMSLER, Affective Literacies. Writing and Multilingualism in the Late Middle Ages, Turnhout, 2011, p. 302 (nous traduisons) : «In fourteenth- and fifteenth-century England, multilingual interactions in English, Anglo-Norman, Latin, and various hybrid languages were the norm rather than the exception, for merchants trading goods, for diplomats carrying information, for preachers, for workers and overseers on the manor, for agents and allies of Richard II and Henry IV, for poets at court and writers of texts in household miscellanies of medical recipes. Often, people were speaking, listening, writing, and reading across languages, for both communication and parody, expression and resistance. English, AngloNorman, and Latin were all living languages at the time. They were often combined at the sentence and lexical levels, but distributed differently and valued differently, unevenly, across the speech community. Only more writing, more play, more countercommentary can resist the work of authoritative language ideology. In performance space and on the manuscript page, creolized Latin reveals linguistic authority to be anything but natural. Rather, there 
Alban Gautier - Université du Littoral Côte d'Opale, HLLI (Unité de recherche sur l'histoire, les langues, la littérature et l'interculturel, EA 4030) - Institut universitaire de France

Jean-Pascal Pouzet - Université de Limoges, CESCM (Centre d'études supérieures de civilisation médiévale, UMR 7302)

are contested textualities and new heterotopes fortified by creole language, deterritorialized utterances, hybrid literate subjects, and multilingual imaginings. » 


\section{Langues d'Angleterre,}

coordonné par Alban Gautier et Jean-Pascal Pouzet

5 Alban Gautier et Jean-Pascal Pouzet

Les langues de

I'Angleterre médiévale : au-delà du bilinguisme

\section{David Trotter}

Peut-on parler de judéoanglo-normand?

Textes anglo-normands en écriture hébraíque

35 Christopher Lucken

Le beau français

d'Angleterre. Altérité de l'anglo-normand et invention du bon usage

57 Aude Mairey John Gower ou le multilinguisme en action

73 Catherine Nall et Daniel Wakelin Le déclin du multilinguisme dans The Boke of Noblesse et son Codicille de William Worcester
Essais et recherches

93 Donatella Nebbiai

Les livres de Jean

Durand († 1416),

"physicien »

et astrologue

119 Lucie Laumonier

En prévision des vieux

jours: les personnes

âgées à Montpellier

à la fin du Moyen Âge
Points de vue

147 François Foronda

Procès politiques:

une manie française?

161 Clément Lenoble

Monnaie, valeur

et citoyenneté chez

Olivi et Eiximenis.

"Moralisation

de l'économie » ou

"économie politique»

médiévale?

181 Notes de lecture

205 Livres reçus 